\title{
Present status of bariatric surgery in Poland
}

\author{
Michał R. Janik, Edward Stanowski, Krzysztof Paśnik \\ Department of General, Oncologic, Metabolic and Thoracic Surgery, Military Institute of Medicine, Warsaw, Poland \\ Videosurgery Miniinv 2016; 11 (1): 22-25 \\ DOI: $10.5114 /$ wiitm.2016.58742
}

\begin{abstract}
Introduction: The first survey of bariatric surgery in Poland was published in 2007. New trends are observed worldwide, and there is a current need to investigate the status of bariatric surgery in Polish institutions. This survey was initiated to gain an overview of Polish bariatric surgery during 2007-2014.

Aim: To analyze the number and types of bariatric procedures performed in Polish institutions in 2014 and to perform a trend analysis from 2007 to 2014.

Material and methods: A questionnaire regarding the numbers and types of bariatric procedures performed between 2007 and 2014 was e-mailed to all members of the Bariatric Society (a branch of the Association of Polish Surgeons) and to 28 surgical departments. Trend analyses from 2007 to 2014 were performed.

Results: Among the surgical departments, $16(57 \%)$ responded. The results showed that 1499 bariatric procedures were performed in Poland in 2014, with 96.4\% done laparoscopically. The highest number was from Masovian Voivodeship. The most commonly reported procedures were laparoscopic sleeve gastrectomy (LSG) (60.7\%), followed by laparoscopic Roux-en-Y gastric bypass (19.2\%), mini gastric bypass (11.1\%), and adjustable gastric banding (7.6\%). Most significant was the rise in prevalence of LSG from 8.0\% to 60.4\% of the total bariatric procedures from 2007 to 2014.

Conclusions: Laparoscopic sleeve gastrectomy is currently the most frequently performed bariatric procedure in Poland. The accuracy of the national survey of procedures would be enhanced if we could create a national registry.
\end{abstract}

Key words: bariatric surgery, trends, Polish survey.

\section{Introduction}

Bariatric surgery is the most effective treatment option for obesity. Its effectiveness in achieving weight loss and fewer co-morbidities was proven in a meta-analysis [1]. Bariatric surgery in Poland began during the 1970s with an open-surgery approach, making the start of this era difficult for both patients and surgeons because of the number of surgical complications. The development of laparoscopy led to dynamic evolution and popularization of bariatric surgery [2]. The first survey of bariatric surgery in Poland was published in 2007 [3], at which time the authors pointed that the most popular procedures were vertical gastric banding (VGB) and adjustable gastric banding (AGB), which constituted about $77 \%$ of all procedures.

Bariatric surgery is still evolving, but since the first Polish articles appeared there have been no others on bariatric surgery in Poland. New trends are observed all over the world [4-8], and there is a current need to investigate the status of bariatric surgery in Polish institutions. Hence, we conducted an overview survey of Polish bariatric surgery during 2007-2014 and report the results here.

\section{Aim}

Our aim was to analyze the number and types of bariatric procedures performed in Polish institutions

\section{Address for correspondence}

Michał R. Janik, Department of General, Oncologic, Metabolic and Thoracic Surgery, Military Institute of Medicine, 128 Szaserów St, 04-141 Warsaw, Poland, phone: +48 602780 960, e-mail: janiken@gmail.com 
in 2014. We also performed a trend analysis from 2007 to 2014 to assess how the choice of procedures has changed over this period.

\section{Material and methods}

A questionnaire evaluating the number and the type of bariatric procedures performed between 2007 and 2014 was administered to all members of the Bariatric Society, which is a branch of the Association of Polish Surgeons. The questionnaire was first administered personally during one of the informal meetings of the Polish Bariatric Society in Warsaw on April 27-28, 2015 and was then e-mailed to all Polish surgical departments that offered bariatric procedures. If this first request was unanswered, further reminders via e-mail and personal contacts were initiated.

\section{Results}

Among the 28 surgical departments that offered bariatric procedures, only 16 returned the questionnaires, giving a response rate of $57 \%$.

\section{Number of procedures performed}

A total of 1499 bariatric procedures were performed in 2014. Among them, $96.4 \%$ were carried out laparoscopically. The highest number of procedures was reported in Masovian Voivodeship. During the period from 2007 to 2014, the total number of procedures per year increased from 176 (2007) to 1499 (2014) (Figure 1).

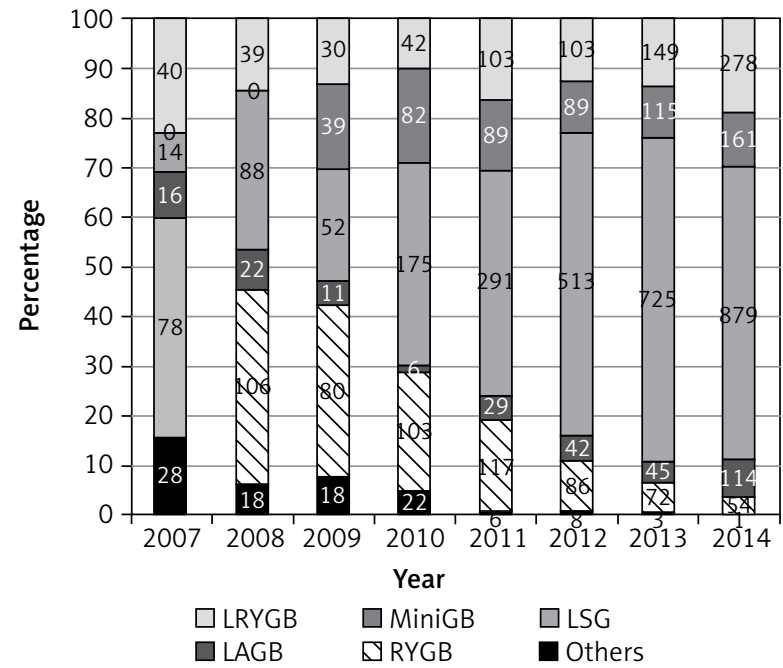

Figure 2. Trend in the numbers and percentages of bariatric procedures performed in Poland during 2007-2014

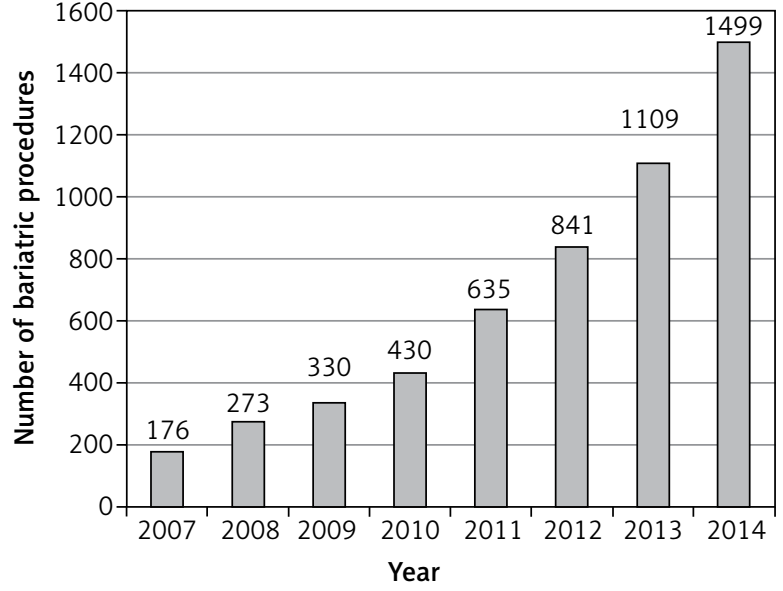

Figure 1. Number of reported bariatric procedures performed in Poland annually during 2007-2014

\section{Types of operation performed}

The most commonly reported procedures in 2014 were laparoscopic sleeve gastrectomy (LSG) (879, $60.7 \%$ ), followed by laparoscopic Roux-en-Y gastric bypass (LRYGB) $(278,19.2 \%)$, mini gastric bypass (MGB) (161, 11.1\%), and AGB (114, 7.6\%) (Figures 2, 3).

\section{Change in trends}

Figures 2 and 3 show the changes in trends over 7 years (2007-2014). Laparoscopic sleeve gastrec-

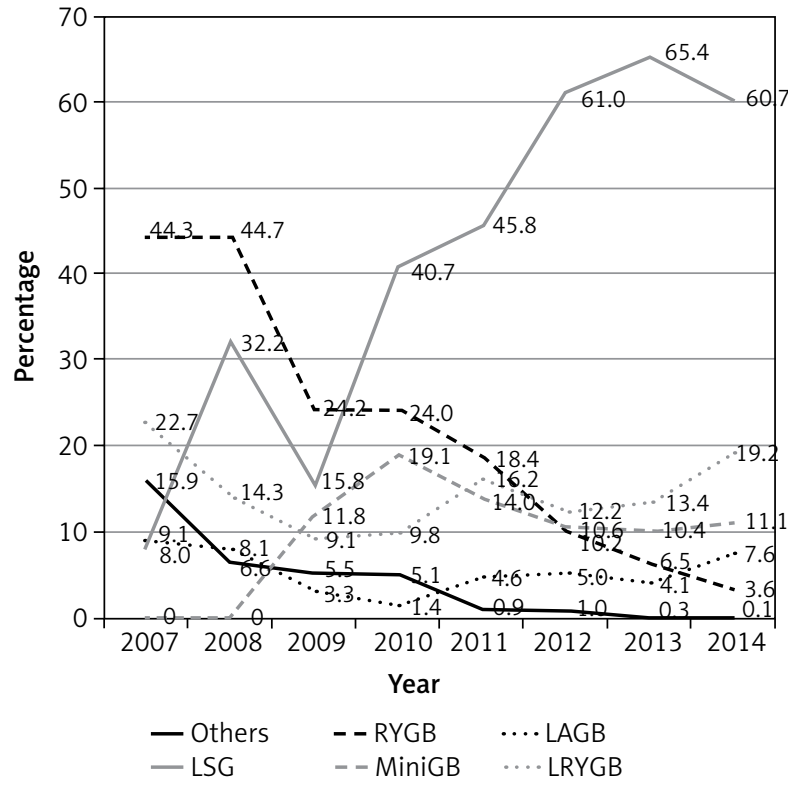

Figure 3. Trend in the percentage of bariatric procedures performed in Poland during 2007-2014 


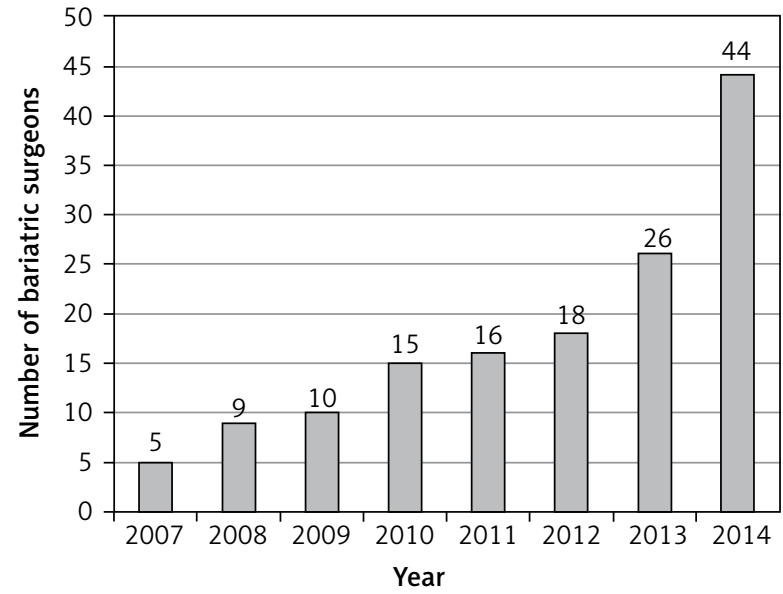

Figure 4. Trend in the number of bariatric surgeons in Poland during 2007-2014

tomy increased significantly from 2009 to 2014 (+42.9\%). At present, LSG represents the most popular bariatric procedure performed in Poland. The reported LRYGB decreased from 2007 to 2010 (-13\%) and then slightly increased up to $19.2 \%$ in 2014 (+9.4\%). In 2009, a new procedure, MGB, was introduced and constituted $11.8 \%$ of all procedures. Mini gastric bypass increased to $19.1 \%$ in 2010 and then dropped to about $10.0 \%$. At present, the proportion of MGBs is stable at $11.1 \%$ in 2014 . The use of laparoscopic adjustable gastric banding (LAGB) is stable at 3.3-8.0\%. We observed a stepwise decrease in open-approach RYGB from $44.7 \%$ in 2008 to $3.6 \%$ in $2014(-41.1 \%)$. There was a significant decrease in other procedures from $15.9 \%$ in 2007 to $0.1 \%$ in 2014 .

\section{Number of bariatric surgeons}

Changes in the total number of bariatric surgeons are shown in Figure 4. We observed a significant increase in the number of bariatric surgeons reported by the centers, from five bariatric surgeons in 2007 to 44 in 2014.

\section{Discussion}

Our survey revealed a continuous increase in the number of bariatric procedures and bariatric surgeons over the years 2007 and 2014. Bariatric surgery is constantly developing. According to the data, laparoscopy is the "gold standard" for bariatric surgery. We observed an "LSG boom" in bariatric surgery. Laparoscopic sleeve gastrectomy outnumbered the rest of the procedures and is now the most popular bariatric procedure in Poland. This trend has also been observed worldwide [8]. The number of LRYGB operations dropped between 2007 and 2009 and then slightly increased to become the second most popular bariatric procedure in Poland. Our data for open-approach RYGB, performed only sporadically, show that it is the least popular procedure. We observed a stepwise decrease in the number of open RYGBs performed from 2007 to 2014. Our survey showed that a new procedure, MGB, was introduced in 2009. Mini gastric bypass represents approximately $10-11 \%$ of the total bariatric procedures annually, making it the third most popular. We found that LAGB is rarely performed.

There has been an increasing incidence of obesity across Europe in recent years. About $15.8 \%$ of Polish adults are obese [9]. Because obesity is associated with co-morbidities such as diabetes and cardiovascular disease, it has a negative impact on general health care $[10,11]$.

Bariatric surgery is the most effective treatment for obesity and related diseases [1, 12]. Bariatric surgery was first performed in Poland during the 1970s, using an open-surgery approach. Hence, it was difficult at the beginning for both patients and surgeons because of the number of surgical complications. However, the development of laparoscopy led to dynamic evolution of this field of surgery [2]. The first Polish survey that focused on bariatric surgery was published in 2007. The authors reported on 614 bariatric procedures in 2005. According to the survey, vertical gastric banding was the most popular bariatric procedure from 1993 to 1997. During the following years, however, this procedure experienced a stepwise decrease in its use. In 2005, there were two popular bariatric procedures: LAGB and RYGB. It should be noted that, at this time, more than half of the RYGBs were performed using an open approach, although the authors reported an increasing tendency to use laparoscopic procedures for bariatric operations [3].

The International Federation for the Surgery of Obesity and Metabolic Disorders (IFSO) reported that 814 bariatric procedures were performed by 20 bariatric surgeons in Poland in 2008 [6]. In 2011, there were 1250 bariatric operations and about 38 bariatric surgeons. LSG was reported to be the most popular bariatric procedure [7]. The latest report, in 2013, showed an increase in the total number of bariatric operations to 1658 in 16 centers. Laparo- 
scopic sleeve gastrectomy was again reported to be the most popular bariatric operation. According to some authors, $96 \%$ of the procedures were performed laparoscopically [8]. Analysis of the reports revealed an increasing trend in the total numbers of procedures and bariatric surgeons. Those results correspond with ours, although the IFSO reported higher numbers of bariatric procedures and surgeons. The differences may be explained by the low response rate in our study, which is the main limitation of our study. We believe that our results are underestimations. We see the need to establish a national registry of bariatric operations. This would allow collection of reliable data concerning the number of procedures, bariatric surgeons, and related complications. Furthermore, collected data regarding complications would be very helpful for increasing the safety of bariatric surgery.

\section{Conclusions}

We observed an increasing trend in the total number of bariatric procedures and bariatric surgeons in Poland from 2007 to 2014. Laparoscopic sleeve gastrectomy is currently the most frequently performed bariatric procedure in Poland. At present, laparoscopy is the gold standard for bariatric surgery. The accuracy of the national survey of procedures would be enhanced if we created a national registry of bariatric procedures.

\section{Conflict of interest}

The authors declare no conflict of interest.

\section{References}

1. Colquitt JL, Pickett K, Loveman E, et al. Surgery for weight loss in adults. Cochrane Database Syst Rev 2014; 8: CD003641.

2. Pardela M, Wiewiora M, Sitkiewicz T, et al. The progress in bariatric surgery. J Physiol Pharmacol 2005; 56: 35-44.

3. Stanowski E, Wyleżoł M, Paśnik K. Laparoscopy in bariatric surgery in Poland - present status. Videosurgery Miniinv 2007; 2: 18-23.

4. Scopinaro N. The IFSO and obesity surgery throughout the world. Obes Surg 1998; 8: 3-8.

5. Buchwald H, Williams SE. Bariatric surgery worldwide 2003. Obes Surg 2004; 14: 1157-64.

6. Buchwald H, Oien DM. Metabolic/bariatric surgery worldwide 2008. Obes Surg 2009; 19: 1605-11.

7. Buchwald H, Oien DM. Metabolic/bariatric surgery worldwide 2011. Obes Surg 2013; 23: 427-36.

8. Angrisani L, Santonicola A, lovino P, et al. Bariatric Surgery Worldwide 2013. Obes Surg 2015; 25: 822-32.
9. Organisation for Economic Co-operation and Development [Internet]. Paris: OECD Obesity update June 2014; (c)2014 [cited 2014 June 26]. Available from: http://www.oecd.org/els/ healthsystems/ Obesity-Update-2014.pdf.

10. Buchwald $H$, Avidor $Y$, Braunwald E, et al. Bariatric surgery: a systematic review and meta-analysis. JAMA 2004; 292: 1724-37.

11. Terranova L, Busetto L, Vestri A, et al. Bariatric surgery: cost-effectiveness and budget impact. Obes Surg 2012; 22: 646-53.

12. Maggard MA, Shugarman LR, Suttorp M, et al. Meta-analysis: surgical treatment of obesity. Ann Intern Med 2005; 142: 547-59.

Received: 22.02.2016, accepted: 14.03.2016. 